\title{
Exercício Físico: Compreendendo as Razões para Prática e seus Desfechos Psicológicos Positivos
}

\author{
Evandro Morais Peixoto ${ }^{1}$ D \\ Universidade São Francisco, Campinas-SP, Brasil
}

\section{RESUMO}

Pesquisas têm evidenciado contribuições do exercício físico na promoção de saúde mental e favorecimento de emoções positivas. Esta pesquisa propõe a avaliação das propriedades psicométricas do Inventário de Razão para o Exercício (REI). Uma amostra de 206 praticantes (idade $=27,31 \pm 8,01,50 \%$ mulheres), respondeu aos instrumentos. Por meio da análise fatorial confirmatória, comparouse os índices de ajustamento de modelos de medidas empregados para o REI na literatura, foram estimados indicadores de precisão e correlação com estado de mindfulness durante o exercício físico. Os resultados indicaram melhores índices de ajustamento para o modelo composto por sete fatores (razões para a prática): controle de peso, condicionamento, humor, atratividade, saúde, tônus e divertimento, todos com bons indicadores de precisão. Verificou-se associação positiva moderada entre indicadores de mindfulness com as razões intrínsecas, humor e divertimento. Conclui-se que a versão brasileira do REI é uma medida adequada das razões para o exercício, correspondente à versão original.

Palavras-chave: psicologia do esporte; psicologia positiva; motivação; saúde mental; validade do teste.

ABSTRACT - Physical Exercise: understanding the reasons for its practice and positive psychological outcomes Studies have shown the role of exercise in promoting mental health and fostering positive emotions. This study proposes the assessment of the psychometric properties of the Reasons for Exercise Inventory (REI). A sample of 206 physical exercise practitioners (mean age 27.31 years $\pm 8.01,50 \%$ women) answered the instruments. The fit indices of different measurement models employed for the REI in the literature were compared through Confirmatory Factor Analysis. Reliability and its correlation with the state of mindfulness during exercise were estimated. The results indicated a better fit for the model composed of seven factors (reasons for exercise): weight control, fitness, mood, health, attractiveness, enjoyment and tone, all with good reliability indicators. Moderate positive associations between mindfulness indicators and intrinsic reasons for exercise: mood and enjoyment were observed. It was concluded that the Brazilian version of the REI is an adequate measure of the reasons for exercise, corresponding to the original version.

Keywords: sport psychology; positive psychology; motivation; mental health; test validly.

RESUMEN - Ejercicio Físico: comprendiendo las razones de la práctica deportiva y sus resultados psicológicos positivos Las investigaciones demuestran las contribuciones del ejercicio físico para la promoción de la salud mental y estimulación de las emociones positivas. Este estudio propone la evaluación de las propiedades psicométricas del Inventario de Razones para el Ejercicio (REI). Una muestra de 206 practicantes (edad $=27.31 \pm 8.01,50 \%$ mujeres) respondió a los instrumentos. A través del Análisis Factorial Confirmatorio, se compararon los índices de ajuste de los modelos de medición utilizados para el REI en la literatura, se estimaron indicadores de precisión y correlación con el estado de mindfulness durante la práctica de ejercicio físico. Los resultados indicaron mejores índices de ajuste para el modelo de siete factores (razones para la práctica): control de peso, entrenamiento, estado de ánimo, atracción, salud, tono muscular y diversión; todos con buenos indicadores de precisión. Se comprobó una relación positiva moderada entre los indicadores de mindfulness y razones intrínsecas, estado de ánimo y diversión. Se puede concluir que la versión brasileña del REI es una medida adecuada de las razones para el ejercicio físico, correspondiendo con la versión original. Palabras clave: psicología del deporte, psicología positiva, motivación, salud mental; validez de test.

De acordo com Seligman (2002), um dos objetivos da Psicologia Positiva é promover as forças e virtudes que ajudem os indivíduos e comunidades a irem além da sobrevivência, possibilitando seu crescimento e desenvolvimento de maneira saudável. Para Faulkner et al. (2015), a atividade física é um comportamento que pode contribuir com esses dois aspectos. Para justificar essa afirmação, os autores destacam que, no nível individual, pesquisas evidenciam a capacidade da atividade física na prevenção doenças mentais, redução dos efeitos de estados negativos (estresse e depressão), e no favorecimento de emoções positivas. No nível social, a atividade física pode ser vista com um importante capital social, uma vez que apresenta a potencialidade de melhorar o nível de saúde física e mental da população, tudo isso com baixos níveis de dispêndio financeiro. Por fim, fazendo alusão ao capítulo de livro Physical Activity as a "Stellar" Positive Psychology Intervention (Hefferon \& Mutrie, 2012), concluem que a atividade física é uma intervenção de grande potencial (estelar) em Psicologia Positiva.

Vale destacar que a compreensão de atividade física apresentada por Faulkner et al. (2015) corresponde 
a qualquer movimento corporal que resulte em gasto de energia acima do nível de repouso. Nesse sentido, o exercício físico pode ser definido como uma subclasse da atividade física, a qual se caracteriza como uma atividade estruturada, comumente supervisionada, e realizada com objetivo de manter ou melhorar o nível de aptidão física ou saúde. Na presente pesquisa, destaca-se a potencialidade do exercício físico como intervenção em Psicologia Positiva, compreendendo-o como uma ferramenta que pode contribuir para que as pessoas tenham uma vida mais gratificante. Nessa direção, a psicologia do exercício tem enfatizado a necessidade de novos investimentos relacionados à investigação dos fenômenos psicológicos que contribuem para uma relação saudável e duradoura com exercício físico, tendo em vista os benefícios dessas atividades na vida cotidiana de seus praticantes, como melhora da aptidão física, promoção de emoções positivas e percepção de bem-estar (Peixoto, Nakano, et al., 2019; Salama-Younes, 2011; Vallerand, 2015).

Pesquisas empíricas têm evidenciado os benefícios psicológicos da prática de exercício físico em amostras representantes de diferentes etapas do desenvolvimento humano. Por meio de uma metanálise, Rebar et al. (2015) avaliaram o potencial antidepressivo e ansiolítico do exercício físico na população de adultos não clínicos. Para tanto, acessaram 92 estudos (correspondentes a 4,310 participantes) que avaliaram o efeito da atividade física/exercício físico na depressão, e 306 estudos (10,755 participantes) que avaliaram os efeitos dessas atividades sobre a ansiedade. Os resultados indicaram que a atividade física/exercício físico teve um efeito de magnitude moderada na redução da depressão, e efeito de baixa magnitude na diminuição da ansiedade (tamanhos de efeitos iguais a 0,50 e $-0,38$, respectivamente), demonstrando a adequação dessas atividades na redução dessas variáveis psicológicas na população de adultos não clínicos.

Edwards et al. (2004) verificaram a associação da prática regular de diferentes tipos de exercício físico (aeróbio, resistência e treinamento de força) e esporte (Hockey) com componentes da percepção de bem-estar físico e psicológico e autoestima em universitários. Os resultados indicaram que os universitários praticantes de exercícios físicos e esportes apresentaram melhores níveis de percepção de bem-estar físico, psicológico e autoestima quando comparados a não praticantes. $\mathrm{Na}$ mesma direção, a população de idosos também tem se beneficiado da prática de exercício, como demonstraram Elavsky et al. (2005) ao realizarem um estudo longitudinal, no período de cinco anos, em que idosos que fizeram parte de um programa de exercício físico no período de seis meses apresentaram melhores níveis de autoeficácia, autoestima, afetos positivos no período de um ano após a realização da intervenção. Os resultados também indicaram melhoras na percepção da qualidade de vida associadas a melhora na autoeficácia e afetos positivos, sugerindo a potencialidade do exercício físico no desenvolvimento da qualidade de vida em longo prazo.

Os resultados de inúmeras pesquisas indicando a associação entre prática de exercício físico e a desenvolvimento de característica psicológica positiva levou importantes instituições, de envergadura internacional, representantes da psicologia do esporte, a publicarem documentos destacando os benefícios do exercício (Associaton for Applied Sport Psychology, n.d; International Society of Sport Psychology, 1992), e tem levado pesquisadores a propor atuação em psicologia do esporte e do exercício físico baseada nos fundamentos da Psicologia Positiva (Brady \& Grenville-Cleave, 2018; Nakano \& Peixoto, 2020; Salama-Younes, 2011). De maneira geral, o que esses autores sugerem é que a psicologia do esporte e do exercício pode se beneficiar da aplicação da Psicologia Positiva, haja vista que muito ainda pode ser explorado em relação as potencialidades da prática de exercícios físicos para a promoção do bem-estar, da saúde mental e do desenvolvimento positivo, bem como em relação a variáveis relacionadas ao engajamento e manutenção dessas atividades ao longo da vida (Brady \& Grenville-Cleave, 2018), como pertencimento social e divertimento.

Dentre os fenômenos psicológicos associados à adesão e à manutenção da prática de exercícios físicos, têm-se destacado as razões para a prática do exercício, uma vez que esta tem se mostrado uma variável que contribui fortemente para o engajamento e manutenção dessas práticas ao longo dos anos. De acordo com Silberstein et al. (1988), as razões para a prática do exercício físico representam diferentes motivações que as pessoas podem apresentar para iniciar e manterem-se praticando. Ter conhecimento das razões pelas quais as pessoas se exercitam pode ajudar os pesquisadores a compreender os fatores motivacionais associados a esse comportamento, o que, por sua vez, pode levar a intervenções e programas de exercícios mais adequados às características dos participantes e, portanto, mais eficazes. Esforços nessa direção tornam-se mais relevantes ao se constatar que, embora sejam amplamente divulgados socialmente os efeitos positivos do exercício físico sobre a saúde física e psicológica, uma esmagadora porcentagem da população nas sociedades industrializadas é sedentária (i.e., cerca de 70\%) ou abandona a prática nos primeiros seis meses (i.e., cerca de $50 \%$ ), dando a entender que esses benefícios não são razões suficientes para que se façam exercícios.

Com objetivo de suprir essa demanda, um modelo teórico sobre as razões para a prática de exercício foi proposto por Silberstein et al. (1988) e era composto de sete diferentes razões que levariam as pessoas a se exercitarem: controle de peso, condicionamento físico, saúde, tônus muscular, atratividade física, humor e divertimento. Esse modelo foi operacionalizado por meio do Inventário de Razões para o Exercício (REI), que é composto por 24 itens e tinha por objetivo a avaliação das sete 
respectivas dimensões proposta pelos autores. Estudos iniciais das propriedades psicométricas do inventário no contexto americano indicou estimativas satisfatórias de consistência interna (alfas de Cronbach entre 0,67 e 81), evidências de validade baseadas na relação com variáveis externas, associação positiva de magnitude moderada entre as razões para o exercício "controle de peso" e controle do "humor" com indicadores de transtornos alimentares. Também foram observadas diferenças significativas entre os escores apresentados por homens e mulheres na dimensão controle de peso, tendo as mulheres apresentado escores mais elevados (Silberstein et al., 1988).

Um estudo baseado na avaliação da estrutura interna do REI foi proposto por Cash et al. (1994) quando, por meio de análise de componentes principais, os autores verificaram a estruturação dos itens em quatro dimensões; a primeira agrupou os itens desenvolvidos para avalição das razões de controle de peso e aparência (ex.: para perder peso, para melhorar minha aparência), na segunda dimensão, agruparam-se as razões de condicionamento físico e saúde (ex.: 24 - Para melhorar minha resistência física, vigor físico, 13 - Para melhorar minha saúde), a terceira dimensão correspondeu às razões voltadas para o controle de humor (ex.: 1 - Para enfrentar a tristeza, depressão, 4 - Para melhorar o meu humor), e a quarta dimensão, denominada socialização quanto a prática de exercícios, tinha como principais razões experiências interpessoais (ex.: 11 - Para socializar com amigos, 20 - Para conhecer novas pessoas). Além disso, dois itens foram excluídos da estrutura fatorial por não se agruparem nas dimensões supracitadas. São eles: 15 - Para melhorar o meu tônus muscular e 23 - Para manter o meu peso corporal.

Outro estudo foi proposto por Strelan et al., (2003), em que foram verificadas estimativas de consistência interna para as sete dimensões originais do instrumento. Com exceção da dimensão controle de peso, foram verificados bons índices da estatística alfa de Cronbach (entre 0,72 a 0,88 ). Contudo, com a exclusão do item 23 - Para manter o meu peso corporal, a dimensão controle de peso teve uma melhora na estimativa de consistência interna de 0,54 para 0,78. Adicionalmente, avaliaram as correlações entre as dimensões a partir das quais basearam uma nova proposta de interpretação do instrumento. Verificaram-se correlações positivas altas entre a dimensão controle de peso e as dimensões tônus muscular e atratividade ( $r^{\prime} s=0,79$ e $\left.0,67, p<0,01\right)$, dessa forma foi sugerido a composição de uma dimensão denominada razões relacionadas à aparência. Correlação alta também foi observada entre as dimensões saúde e condicionamento físico $(r=0,83, p<0,01)$, e entre divertimento e humor $(r=0,6, p<0,01)$, levando a composição de outras duas dimensões, a saber: razões relacionadas à saúde/condicionamento e divertimento/humor.

Nessa nova configuração, o instrumento apresentou bons indicadores de precisão, alfas entre 0,85 e 0,91, bem como resultados de associação com outras variáveis externas (auto-objetificação, satisfação corporal, estima corporal e autoestima) coerentes com a expectativa teórica. Assim foram verificadas correlações positivas entre razões relacionadas à aparência e auto-objetificação $(r=0,78)$, e correlações negativas de magnitude moderada a alta com as variáveis satisfação corporal $(-0,57)$, estima corporal $(-0,78)$ e autoestima $(-0,55)$. Por outro lado, as razões relacionadas à saúde/condicionamento se relacionaram negativamente com auto-objetificação $(-0,73)$ e positivamente com satisfação corporal $(0,61)$, estima corporal $(0,68)$ e autoestima $(0,50)$. Movimento semelhantes foi observado com a dimensão divertimento/humor, em que foram observadas as seguintes correlações: $-0,56 ; 0,47 ; 0,50$ e 0,45 , respectivamente. Ressalta-se que todas as estimativas foram significativas, com $p<0,01$ (Strelan et al., 2003).

A estrutura interna proposta para o REI a partir de Strelan et al. (2003) tem sido adotada por diferentes autores que tem por objetivo avaliar as razões para a prática de exercício físico (Cox, Ullrich-French, French, et al., 2016; Cox, Ullrich-French, Cole, et al., 2016). Destacase também que o instrumento foi adotado em diferentes culturas, como a americana (Luu, 2014, Strömmer et al., 2015), a chinesa (Yan, 2008; Yan et al., 2014) e também na cultura brasileira (Peixoto, Palma et al., 2019). Contudo, estudos de evidências de validade que comparem os diferentes modelos ainda são escassos em ambos os contextos, nacional e internacional.

Com base no exposto, esta pesquisa teve como principais objetivos estimar evidências de validade com base na estrutura interna, na relação com variável externa e precisão do REI frente a uma amostra de praticantes de exercícios físicos provenientes do interior do estado de Pernambuco. Para tanto, propõe-se a comparação dos indicadores de adequação dos diferentes modelos de medida difundidos na literatura: composto por sete (Silberstein et al., 1988), quatro (Cash et al., 1994) e três dimensões (Strelan et al., 2003). Também se propõe a estimação da correlação entre o REI e a Escala de Mindfulness para Atividade Física (Cox, Ullrich-French, French, et al., 2016), tendo como hipótese a associação positiva entre as razões mais internas (autodeterminadas) para a prática de esporte.

Com esta pesquisa, espera-se contribuir para o preenchimento de uma importante lacuna da psicologia do esporte do exercício brasileira, a escassez de investimento científico na compreensão do envolvimento da população geral com o exercício físico e, portanto, de instrumentos de medida disponíveis aos pesquisadores e profissionais de área, haja vista que grande parte desses investimentos se encontra direcionados ao alto rendimento esportivo, ficando restrito muitas vezes a um número limitado de beneficiados (Côté \& Vierimaa, 2014; Jayanthi et al., 2012). Além disso, este estudo oferece uma contribuição à literatura internacional ao propor a comparação dos 
modelos de medida empregados na literatura, haja vista que até o momento não foram encontradas pesquisas com esse fim.

\section{Método}

\section{Participantes}

A amostra foi composta por 206 praticantes de diferentes modalidades de exercícios físicos (corrida, musculação, crossfit, entre outras) de ambos os sexos (50\% mulheres), com idades que variavam entre 18 e 62 anos $(M=27,31 \pm 8,01)$. Quanto ao estado civil, 68\% se autodeclararam solteiros, $28,6 \%$ casados e 3,4\% divorciados. Em relação ao nível de escolaridade, $42,2 \%$ apresentavam nível universitário incompleto; $37,9 \%$ universitário completo; $14,1 \%$ ensino médio completo; $3,9 \%$ ensino médio incompleto e $2 \%$ ensino fundamental completo.

\section{Instrumentos}

Reasons for Exercise Inventory REI (Silberstein et al., 1988). Propõe a mensuração das razões internas ou externas para a prática de exercício físico por meio de 24 itens respondidos em escala do tipo Likert de sete pontos variando entre $1=$ Discordo fortemente e $7=$ Concordo fortemente. Estudo referente a propriedades psicométricas desse instrumento, conforme apresentado no tópico Introdução, tem suportado a avaliação de sete (Silberstein et al., 1988), quatro (Cash et al., 1994) ou três dimensões (Strelan et al., 2003). Em todos os estudos, foram obtidos bons indicadores de precisão, alfa de Cronbach superiores a 0,7 .

Escala de estado de Mindfulness para Atividade Física SMS-PA (Cox, Ullrich-French, French, et al., 2016). O instrumento tem por objetivo a avaliação do estado de mindfulness durante a prática de exercício físico e esporte. É composto por 12 itens teoricamente organizados em uma estrutura bifactor composta por dois fatores específicos, mindfulness mental e mindfulness físico e um fator geral. Estudos referentes às propriedades psicométricas encontraram evidências empíricas que sustentam o modelo bifactor para versão americana (Cox, UllrichFrench, French, et al., 2016), espanhola (Ullrich- French et al., 2017) e brasileira (Peixoto, Palma et al., 2019) da SMS-PA, bem como bons níveis de precisão, coeficientes alfas de Cronbach superiores a 0,80 .

Questionário Sociodemográfico. $\mathrm{O}$ instrumento permitiu acesso às principais características dos participantes, como sexo, idade, tipo de exercício realizado, tempo de experiência com exercício e nível de escolaridade.

\section{Procedimentos}

\section{Considerações Éticas}

O contato com a amostra e, portanto, a aplicação dos instrumentos ocorreu após a aprovação do projeto pelo Comitê de Ética e Pesquisa da Pontifícia Universidade Católica de Campinas, sob número de protocolo CAAE 38072214.8.0000.5481. O contato com os participantes da pesquisa se deu em praças esportivas, parques, academia de ginásticas e crossfit localizadas no interior do estado de Pernambuco. Os instrumentos foram administrados de forma coletiva, antecedido da assinatura do Termo de Consentimento Livre e Esclarecido TCLE. Ao participante era garantido o direito de ser informado sobre os objetivos e procedimentos da pesquisa, sigilo sobre os dados coletados e a possibilidade de desistir de sua participação em qualquer momento da realização da pesquisa, atendendo à Resolução 466/2012 do Conselho Nacional de Saúde.

\section{Análise de Dados}

Para comparação dos modelos de medidas observados na literatura para REI, empregou-se a análise fatorial confirmatória (AFC) com método de estimação Robust Weighted Least Square (WLSMV), devido à adequação do método quando as variáveis em análise são ordinais, como no caso do sistema de resposta em estilo Likert de 7 pontos empregado no instrumento. Dessa forma, os modelos foram testados e comparados a partir dos índices de ajustamento recomendados por Muthén e Muthén (2012): WLSMV $\chi^{2}, g l$, CFI, TLI e RMSEA, tendo como referência os valores de indicadores de ajustamento dos modelos aceitáveis: $\chi^{2} / g l<5$; CFI e TLI $>0,90$ e RMSEA $<0,08$. Tais análises foram realizadas com o apoio do pacote estatístico Mplus 7.11 (Muthén \& Muthén, 2012). Para avaliação da precisão, empregou-se análise de consistência interna por meio dos coeficientes alfa de Cronbach e ômega de McDonald, tendo como referências valores iguais ou superiores a 0,70 .

\section{Resultados}

Para responder ao primeiro objetivo da presente pesquisa, comparou-se os índices de ajuste dos modelos com três, quatro e sete fatores. Os resultados são apresentados na Tabela 1.

A partir dos resultados apresentados na Tabela 1, pode-se inferir que apenas o modelo original do instrumento proposto por sete fatores apresentou índices de ajuste considerados adequados. Nessa direção, a análise DIFTEST indicou que esse modelo apresentou índices de ajuste significativamente melhores quando comparado aos modelos compostos por quatro e três fatores. Outras informações desse modelo são apresentadas na Tabela 2. Onde se verificam as cargas fatoriais dos itens em seus respectivos fatores, estimativas de correlação entre eles e índices de consistência interna. 
Tabela 1

Índices de Ajustamentos dos Modelos com Três, Quatro e Sete Fatores do REI

\begin{tabular}{cccccccc}
\hline Modelos & $\chi^{2}$ & $\mathrm{df}$ & $\chi^{2} / \mathrm{df}$ & CFI & TLI & RMSEA & RMSEA IC90\% \\
\hline Três fatores & 1172,845 & 249 & 4,71 & 0,729 & 0,700 & 0,138 & $0,130-0,146$ \\
Quatro fatores & 1035,231 & 246 & 4,21 & 0,768 & 0,74 & 0,129 & $0,121-0,127$ \\
Sete fatores & 576,67 & 231 & 2,50 & 0,915 & 0,897 & 0,079 & $0,069-0,090$ \\
\hline
\end{tabular}

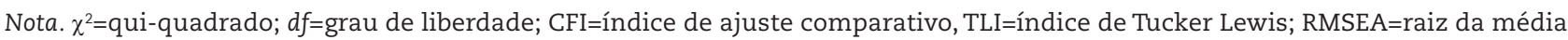
dos quadrados dos erros de aproximação; IC=intervalo de confiança

Tabela 2

Modelo Fatorial Confirmatório do REI

\begin{tabular}{|c|c|c|c|c|c|c|c|}
\hline \multirow{2}{*}{ Itens } & \multicolumn{7}{|c|}{ Fatores } \\
\hline & Cont.Peso & Cond. & Humor & Atrat. & Saúde & Tônus & Divert. \\
\hline 05 & 0,804 & & & & & & \\
\hline 21 & 0,741 & & & & & & \\
\hline 23 & 0,634 & & & & & & \\
\hline 15 & & 0,745 & & & & & \\
\hline 16 & & 0,780 & & & & & \\
\hline 22 & & 0,688 & & & & & \\
\hline 24 & & 0,830 & & & & & \\
\hline 01 & & & 0,706 & & & & \\
\hline 04 & & & 0,719 & & & & \\
\hline 08 & & & 0,853 & & & & \\
\hline 17 & & & 0,845 & & & & \\
\hline 06 & & & & 0,727 & & & \\
\hline 11 & & & & 0,849 & & & \\
\hline 20 & & & & 0,885 & & & \\
\hline 02 & & & & & 0,695 & & \\
\hline 13 & & & & & 0,851 & & \\
\hline 14 & & & & & 0,893 & & \\
\hline 19 & & & & & 0,805 & & \\
\hline 09 & & & & & & 0,834 & \\
\hline 12 & & & & & & 0,919 & \\
\hline 18 & & & & & & 0,791 & \\
\hline 03 & & & & & & & 0,691 \\
\hline 07 & & & & & & & 0,832 \\
\hline 10 & & & & & & & 0,911 \\
\hline Cont.Peso & 1 & & & & & & \\
\hline Cond. & 0,547 & 1 & & & & & \\
\hline Humor & 0,428 & 0,580 & 1 & & & & \\
\hline Atrat. & 0,396 & 0,408 & 0,391 & 1 & & & \\
\hline Saúde & 0,476 & 0,858 & 0,640 & 0,388 & 1 & & \\
\hline Tônus & 0,439 & 0,446 & 0,275 & 0,298 & 0,255 & 1 & \\
\hline Diver & 0,501 & 0,727 & 0,270 & 0,088 & 0,373 & 0,756 & 1 \\
\hline Alfa & 0,763 & 0,883 & 0,819 & 0,822 & 0,849 & 0,862 & 0,832 \\
\hline Ômega & 0,777 & 0,886 & 0,827 & 0,838 & 0,861 & 0,865 & 0,833 \\
\hline
\end{tabular}

Nota. Cont. Peso=Controle de peso; Cond.=condicionamento; Atrat.=atratividade; Diver.=divertimento

A partir dos resultados apresentados na Tabela 2 , observa-se que as cargas fatoriais dos itens em seus respectivos fatores variaram entre 0,634 (item 23 - Para manter o meu peso corporal - correspondente ao fator Controle de peso) e 0,919 (item 12 - Para melhorar a minha aparência correspondente ao fator Tônus). Tais resultados sugerem a 
adequação dos itens, indicando que os conteúdos apresentados podem ser considerados bons representantes de seus respectivos fatores, haja vista a indicação obtida por meio da carga fatorial de que entre 49,19\% e 84,46\% da variância única dos itens foram preditas por esses fatores.

$\mathrm{Na}$ segunda parte da Tabela 2, verificam-se os indicadores de correlação entre os fatores que compõem o REI, os quais variaram entre ausência de correlação $r=0,088$ (Divertimento x Atratividade) a correlações de alta magnitude $r=0,858$ (Saúde X Condicionamento) e 0,756 (Divertimento x Tônus). Adicionalmente, grande parte das correlações entre os fatores apresentou magnitudes moderadas. Por fim, são apresentados os indicadores de precisão, estimativas dos coeficientes alfa de Cronbach, que variaram entre 0,763 (Controle de peso) e 0,883 (Condicionamento) e ômega de McDoland, os quais variaram entre 0,777 (Controle de peso) e 0,886 (Condicionamento), o que sugere que os fatores apresentam bons níveis de precisão e, portanto, níveis aceitáveis de erro associado a mensuração dos conteúdos propostos.

A última etapa da pesquisa consistiu em estimar as correlações entre os escores apresentados pelos participantes no REI e na SMS-PA. Os resultados são apresentados na Tabela 3, onde se destacam as correlações positivas, de baixa magnitude e significativas entre o fator Mindfulness mental com os fatores Condicionamento, Saúde e Controle de peso, as correlações positivas, de magnitudes moderadas e significativas entre o fator Mindfulness mental e os fatores Humor e Divertimento, bem como a relativa ausência de correlação entre a subescala Mindfulness físico e os fatores que compõem o REI. Por fim, observa-se que o Mindfulness geral se relacionou de maneira positiva e de baixa magnitude com as razões para exercício ligadas ao Controle de peso, Saúde, Condicionamento e, na mesma direção, mas com magnitude moderada com as razões relacionadas ao Divertimento e Humor.

Tabela 3

Correlação entre o REI e SMS-PA

\begin{tabular}{lccccccc}
\hline \multicolumn{1}{c}{ Mindfulness } & Cont. Peso & Condic. & Humor & Atratividade & Saúde & Tônus & Divertimento \\
\hline Mental & $0,193^{* *}$ & $0,145^{*}$ & $0,328^{* *}$ & 0,079 & $0,160^{*}$ & 0,111 & $0,290^{* *}$ \\
Físico & 0,019 & 0,102 & 0,015 & 0,060 & 0,065 & $-0,025$ & 0,037 \\
Geral & $0,154^{*}$ & $0,172^{*}$ & $0,268^{* *}$ & 0,093 & $0,155^{*}$ & 0,076 & $0,242^{* *}$ \\
\hline
\end{tabular}

Nota. Cont. Peso=Controle de peso; Cond.=condicionamento; Atrat.=atratividade; Diver.=divertimento; ${ }^{* *} p<0,01$; ${ }^{*} p<0,05$

\section{Discussão}

Esta pesquisa teve como principal objetivo estimar evidências de validade com base na estrutura interna, relação com variáveis externa e precisão para a versão brasileira do REI, um instrumento tradicional, utilizado internacionalmente para a avaliação das razões para a prática do exercício físico. Para tanto, comparou-se diferentes modelos de medidas empregados na literatura: desde aquele proposto originalmente por Silberstein et al. (1998), composto por sete fatores, até modelos sugeridos posteriormente, como o de quatro (Cash et al., 1994) e três fatores (Strelan et al., 2003). Estimou-se associação entre o REI e a SMS-PA, e coeficientes de consistência interna. Tais esforços objetivaram o preenchimento de uma lacuna importante na psicologia do exercício físico, em especial no contexto brasileiro, quanto à necessidade de instrumentos psicométricos cujas propriedades psicométricas sejam conhecidas, e que a interpretações dos escores desses instrumentos tenham como base um argumento de validade construído a partir de pesquisas empíricas sucessivas (American Educational Research Association, American Psychology Association \& National Council on Measurement in Education [AERA, APA \& NCME], 2014).
Os resultados obtidos por meio da AFC indicaram nível de ajustamento significativamente superior para o modelo composto por sete fatores, corroborando a proposta que deu origem ao desenvolvimento do REI (Silberstein et al., 1998). Tais resultados indicam que, mesmo havendo correlações de alta magnitude entre alguns pares de fator (Saúde X Condicionamento e Divertimento x Tônus), há uma melhor indicação de ajustamento quando mantidos os fatores separadamente. Isso vai de encontro aos procedimentos realizados por Cash et al. (1994) ao estabelecer uma única dimensão a partir do agrupamento dos fatores Saúde e Condicionamento, bem como da proposta de Strelan et al. (2003), que a partir da análise de correlação entre as dimensões propuseram a estrutura interna composta por apenas três fatores. Os resultados obtidos na presente pesquisa lançam luz à discussão em relação à estrutura interna do REI e ao propor a avaliação de evidências de validade a partir da comparação desses modelos.

Nesse sentido, vale destacar as nuances dos procedimentos estatísticos e características das amostras disponíveis às diferentes pesquisas. Os resultados encontrados por Cash et al. (1994) foram estimados a partir da Análise de Componentes Principais, tendo como base 
matrizes de correlação de Pearson estimadas em uma amostra composta por 101 mulheres praticantes de exercício. Estudos metodológicos vêm apontando a fragilidade desse procedimento na estimativa da estrutura interna de instrumentos psicológicos, haja vista que esse método não considera variáveis latentes ao estimar a estrutura de covariâncias das variáveis observadas, nesse caso, itens de um teste. Ao contrário da análise fatorial, que busca a compreensão da influência da variável latente na variabilidade das variáveis observadas ao considerar apenas a parcela de variância compartilhada entre os itens na estimação da solução fatorial (Costello \& Osborne, 2005).

Outras críticas se direcionam à utilização de correlação de Pearson para estimativa de estrutura interna de itens respondidos por meio de escala do tipo Likert (variáveis ordinais), uma vez que esse método se destina a análise de variáveis contínuas com distribuição normal, mostrando mais adequado o uso de correlações policóricas (Lara \& Alexis, 2014). Contudo, há de se ressaltar o poder computacional disponível aos pesquisadores atualmente, bem como acessibilidade aos softwares que trazem esses procedimentos implementados, tornando possível a avaliação desses instrumentos por meio de métodos vistos atualmente como mais adequados.

Já a proposta do modelo de medida oferecida por Strelan et al. (2003) foi baseada apenas na avaliação de correlações entre fatores, tendo contado com uma amostra de 104 mulheres. Embora essa estrutura tenha sido empregada em diferentes estudos (Cox, Ullrich-French, French, et al., 2016; Peixoto, Palma et al., 2019), pode-se atribuir uma certa fragilidade a esse procedimento uma vez que existem procedimentos estatísticos específicos para esse fim, como análise fatorial confirmatória, por exemplo (Brown, 2015). Ainda em relação à estrutura interna observada na presente pesquisa, destacam-se as cargas fatoriais elevadas apresentada pelos itens, mesmo aqueles que tiveram a indicação de serem excluídos em estudos anteriores, como os itens 15 - Para melhorar o meu tônus muscular e 23 - Para manter o meu peso corporal (Cash et al., 1994; Strelan et al., 2003), apresentaram-se como bons representantes dos respectivos construtos, razões para práticas do exercício ligadas a Tônus muscular e Controle de peso com cargas fatoriais iguais a 0,745 e 0,634 .

Por fim, aponta-se que algumas características da amostra podem ter contribuído para os resultados observados na presente pesquisa. Ao contar com participantes de ambos os sexos, pôde-se alcançar número amostral suficiente para realização de AFC e estimação de parâmetros confiáveis, ou seja, número de participantes superior a 200 (Myers et al., 2011; Marsh, 2007). Adicionalmente, pôde-se representar mais adequadamente o universo de praticantes de exercícios físicos no contexto brasileiro e, portanto, avaliar a potencialidade do instrumento em ser empregado em pesquisas futuras que objetivam a avaliação dos motivos para a prática de exercícios físicos em amostras compostas por mulheres e homens. Contudo, sugere-se que futuros estudos possam acessar amostras maiores e diversificadas em relação às características dos participantes, como sexo, idade e tipo de exercício praticado, que possibilitem a avaliação da estabilidade do modelo de medida em função da maior variabilidade nos escores dos respondentes.

Em relação às evidências de precisão da REI, os resultados obtidos por meio dos coeficientes alfa de Cronbach e ômega de McDonald são classificáveis como bons, alfa de Cronbach superiores a 0,763 e ômega superiores a 0,777 (Tabachnick \& Fidell, 2012) e coerentes com os resultados observados na proposta original do instrumento (Silberstein et al., 1988) e com os resultados de pesquisas que avaliaram a precisão do modelo composto por sete fatores (Yan, 2008; Yan et al., 2014). Esses resultados sugerem novas evidências quanto a capacidade desse instrumento em avaliar os construtos psicológicos de interesse com uma margem de erro aceitável. Segundo Primi (2012), nenhuma medida psicológica é livre de erro, contudo suas limitações devem ser conhecidas para não colocar em risco a avaliação do construto alvo. Assim, pode-se considerar que o nível de erro observado na modelo de medida com sete fatores do REI assegura o emprego desse modelo em futuras pesquisas.

O estudo das correlações entre as diferentes razões para exercício físico e indicadores de mindfulness indicaram evidências de validade REI com base na relação com outras variáveis. Em acordo com essa expectativa teórica, observou-se correlações positivas moderadas entre Mindfulness mental e Mindfulness geral com as razões para a prática do exercício mais interna (intrínseca), aquelas relacionadas com Divertimento e Humor, corroborando evidências empíricas de associação positiva com motivação autônoma. Segundo Cox, Ullrich-French, French, et al. (2016), o estado de mindfulness pode ser considerado um mecanismo psicológico positivo de suporte a experiência durante a atividade esportiva, tornando-se assim um mecanismo mental do comportamento esportivo autodeterminado que, em acordo com a teoria da autodeterminação (Ryan \& Deci, 2007), contribui para a manutenção da prática do exercício ao longo da vida.

Considerando a definição de mindfulness como a consciência do momento presente, incluindo reconhecimento e aceitação das sensações e sentimentos sem elaborações ou julgamentos, abarcando a consciência dos estímulos como estados mentais (pensamentos e sentimentos) e sensações físicas (tensão muscular ou postura corporal) no momento em que elas ocorrem, faz sentido teórico as observações de correlações de baixa magnitude observada entre mindfulness mental e geral com as razões mais externas (extrínsecas) para prática do exercício, aquelas voltadas para o Condicionamento, Saúde e Controle de peso, uma vez que pessoas que praticam exercícios motivadas por razões externas tendem 
a não experimentar tais estados (Cox, Ullrich-French, French, et al., 2016; Cox et al., 2017).

A partir dos resultados observados na presente pesquisa, pode-se afirmar que os objetivos estabelecidos inicialmente foram satisfatoriamente alcançados, pois se demonstrou diferentes evidências de validade e precisão para o REI quanto à avaliação das diferentes razões para a prática do exercício físico, por meio de um modelo composto por sete fatores. Destaca-se que o uso da AFC possibilitou levantamento de características da escala não verificadas nos estudos anteriores, a comparação de diferentes modelos empregados na literatura, trazendo contribuições para melhor compreensão das potencialidades do instrumento. Além disso, caracteriza-se como uma importante contribuição ao cenário nacional que conta com poucos instrumentos voltados à compreensão das razões para a prática do exercício físico. Ressalta-se tratar de um instrumento tradicional e amplamente utilizado no cenário internacional, e a realização de estudos dessa natureza possibilita o diálogo entre as pesquisas realizadas no contexto brasileiro com aquelas realizadas internacionalmente.

Por fim, destacam-se alguns limites da presente pesquisa que se baseia em uma amostra por conveniência, oriunda de uma região específicas do país, Nordeste. Portanto, sugere-se cautela na generalização dos resultados obtidos, bem como a realização de novos estudos que objetivem estimar outras evidências de validade do REI com amostras mais representativas em relação a outras regiões do país. O incremento da amostra também possibilitará estudos de invariância dos parâmetros do modelo de medida em função de sexo, tipo de exercício praticado e outras variáveis de interesse de futuros pesquisadores, possibilitando o conhecimento de outras potencialidades e possíveis fragilidades do instrumento.

\section{Agradecimentos}

Não há menções.

\section{Financiamento}

A presente pesquisa não recebeu nenhuma fonte de financiamento sendo custeada com recursos dos próprios autores.

\section{Contribuições dos autores}

Evandro Morais Peixoto foi o responsável por toda a elaboração do manuscrito. Incluindo as etapas de redação inicial do estudo - conceitualização, investigação, visualização, análise dos dados, e redação final do trabalho - revisão e edição.

\section{Disponibilidade dos dados e materiais}

Todos os dados e sintaxes gerados e analisados durante esta pesquisa serão tratados com total sigilo devido às exigências do Comitê de Ética em Pesquisa em Seres Humanos. Porém, o conjunto de dados e sintaxes que apoiam as conclusões deste artigo estão disponíveis mediante razoável solicitação ao autor principal do estudo

\section{Conflito de interesses}

Os autores declaram que não há conflitos de interesses.

\section{Referências}

American Educational Research Association, American Psychology Association \& National Council on Measurement in Education (2014). Standards for educational and psychological testing. American Educational Research Association.

Associaton for Applied Sport Psychology. (n.d.). Psychological Benefits of Exercise. https://appliedsportpsych.org/resources/health-fitnessresources/psychological-benefits-of-exercise/

Brady, A., \& Grenville-Cleave, B. (2018). Positive Psychology in Sport and Physical Activity: An introduction. Routledge.

Brown, T. A. (2015). Confirmatory factor analysis for applied research. Guilford Press.

Cash, T. F., Novy, P. L., \& Grant, J. R. (1994). Why do women exercise? Factor analysis and further validation of the Reasons for Exercise Inventory. Perceptual and Motor Skills, 78(2), 539-44. https://pubmed.ncbi.nlm.nih.gov/8022678/

Conselho Nacional de Saúde. (2012). Resolução 466/2012. https://conselho.saude.gov.br/resolucoes/2012/Reso466.pdf

Côté, J., \& Vierimaa, M. (2014). The developmental model of sport participation: 15 years after its first conceptualization. Science E Sports, 29, S63-S69. https://doi.org/10.1016/j.scispo.2014.08.133

Costello, A. B., \& Osborne, J. W. (2005). Best Practices in Exploratory Factor Analysis: Four recommendations for getting the most from your analysis. Practical Assessment, Research, and Evaluation, 10(7), 1-9. Recuperado de https://scholarworks.umass.edu/cgi/viewcontent. cgi? article $=1156 \&$ context $=$ pare

Cox, A. E., Ullrich-French, S., Cole, A. N., \& D'Hondt-Taylor, M. (2016). The role of state mindfulness during yoga in predicting selfobjectification and reasons for exercise. Psychology of Sport and Exercise, 22, 321-327. https://doi.org/10.1016/j.psychsport.2015.10.001

Cox, A. E., Ullrich-French, S., \& French, B. F. (2016). Validity Evidence for the State Mindfulness Scale for Physical Activity. Measurement in Physical Education and Exercise Science, 20(1), 38-49. https://doi.org/10.1080/1091367X.2015.1089404

Cox, A. E., Ullrich-French, S., Howe, H. S., \& Cole, A. N. (2017). A pilot yoga physical education curriculum to promote positive body image. Body Image, 23, 1-8. https://doi.org/10.1016/j.bodyim.2017.07.007

Edwards, D. J., Edwards, S. D., \& Basson, C. J. (2004). Psychological Well-Being and Physical Self-Esteem in Sport and Exercise. International Journal of Mental Health Promotion, 6(1), 25-32. doi:10.1080/14623730.2004.9721921 
Elavsky, S., McAuley, E., Motl, R. W., Konopack, J. F., Marquez, D. X., Hu, L., Jerome., G. J., Diener, E. (2005). Physical activity enhances long-term quality of life in older adults: Efficacy, esteem, and affective influences. Annals of Behavioral Medicine, 30(2), 138-145. https:// doi.org/10.1207/s15324796abm3002 6

Faulkner, G., Hefferon, K., \& Mutrie, N. (2015). Positive Psychology in Motion through Physical Activity. Em S. Joseph (Ed), Positive Psychology in Practice: promoting human flourishing in work, health, education, and everyday life (pp. 207-222). John Wiley \& Sons Inc.

Hefferon, K., \& Mutrie, N. (2012). Physical activity as a "stellar" positive psychology intervention. Em E. O. Acevedo (Ed.), The Oxford handbook of exercise psychology (pp. 117-130). Oxford University Press.

International Society of Sport Psychology (1992). Physical activity and psychological benefits: A position statement from the international society of sport psychology. Journal of Applied Sport Psychology, 4(1), 94-98. https://doi.org/10.1080/10413209208406452

Jayanthi, N., Pinkham, C., Dugas, L., Patrick, B., \& LaBella, C. (2012). Sports Specialization in Young Athletes. Sports Health: A Multidisciplinary Approach, 5(3), 251-257. doi:10.1177/1941738112464626

Lara, D., \& Alexis, S. (2014). ¿Matrices policóricas/tetracóricas o matrices pearson? Un estudio metodológico. Revista Argentina de Cienciasdel Comportamiento, 6(1), 39-48. https://revistas.unc.edu.ar/index.php/racc/article/view/6357

Luu, M. (2014) "Perspiration and Motivation: An Examination of Body Image and Exercise". [Master's thesis, San José State University]. https://scholarworks.sjsu.edu/etd_theses/4474

Marsh, H. W. (2007). Application of confirmatory factor analysis and structural equation modeling in sport and exercise psychology. Em G. Tenenbaum \& R. C. Eklund (Eds.), Handbook of sport psychology (pp. 774-798). Wiley.

Myers, N. D., Ahn, S., \& Jin, Y. (2011). Sample size and power estimates for a confirmatory factor analytic model in exercise and sport: A Monte Carlo approach. Research Quarterly for Exercise and Sport, 82, 412-423. https://doi.org/10.5641/027013611X13275191443621

Muthén, L. K., \& Muthén, B. O. (2012). Mplus user's guide (7th ed.). Muthén \& Muthén. https://www.statmodel.com/download/usersguide/ Mplus\%20user\%20guide\%20Ver_7_r3_web.pdf

Nakano, T. C., \& Peixoto, E. M. (2020).

Peixoto, E. M., Nakano, T. C., Castillo, R. A., O., Pestillo, L., \& Balbinotti, M. A. A. (2019). Passion Scale: Psychometric Properties and Factorial Invariance via Exploratory Structural Equation Modeling (ESEM). Paidéia, 29, e2911. https://doi.org/10.1590/19824327e2911

Peixoto, E. M., Palma, B. P., França-Torres, V. C., Oliveira-Silva, K. N., Farias, R. P., \& Monteiro, V. T. L. (2019). Cross-cultural adaptation and validity evidence of the Brazilian version of the State Mindfulness Scale for Physical Activity (SMS-PA). Journal of Physical Education E Sport, 19(1). https://doi.org/10.7752/jpes.2019.01087

Primi, R. (2012). Psicometria: Fundamentos matemáticos da Teoria Clássica dos Testes. Avaliação Psicológica, 11(2), 297-307. http://pepsic. bvsalud.org/scielo.php?script=sci_arttext\&pid $=$ S1677-04712012000200015\&lng $=$ pt\&tlng $=$ pt.

Ryan, R. M., \& Deci, E. L. (2007). Active human nature: Self-determination theory and the promotion and maintenance of sport, exercise, and health. In M. S. Hagger \& N. Chatzisarantis (Eds.), Intrinsic motivation and self-determination in exercise and sport (pp. 1-19). Human Kinetics.

Rebar, A. L., Stanton, R., Geard, D., Short, C., Duncan, M. J., \& Vandelanotte, C. (2015). A meta-analysis of the effect of physical activity on depression and anxiety in non-clinical adult populations. Health Psychology Review, 9(3), 366-378. https://doi.org/10.1080/1743719 9.2015.1022901

Salama-Younes, M. (2011). Towards a Positive Sport Psychology: A Prospective Investigation in Physical Practice. World Journal of Sport Sciences, 4(2), 104-115. https://pdfs.semanticscholar.org/1b69/f8da96b424c1ba2a3ba288534e1a9f4e2a19.pdf

Seligman, M. E. P. (2002). Positive psychology, positive prevention and positive therapy. Em C. R. Snyder \& S. J. Lopez (Eds.), Handbook of positive psychology (pp. 3-9). Oxford University Press.

Silberstein, L. R., Striegel-Moore, R. H., Timko, C., \& Rodin, J. (1988). Behavioral and psychological implications of body dissatisfaction: Do men and women differ? Sex Roles, 19, 219-232. https://doi.org/10.1007/BF00290156

Strelan, P., Mehaffey, S. J., \& Tiggemann, M. (2003). Self objectification and esteem in young women: The mediating role of reasons for exercise. Sex Roles, 48, 89-95. https://doi.org/10.1023/A:1022300930307

Strömmer, S. S., Ingledew, D. K., \& Markland, D. (2015). Development of the Exercise Motives and Gains Inventory. Measurement in Physical Education and Exercise Science, 19, 53-68. https://doi.org/10.1080/1091367X.2015.1036162

Tabachnick, B. G., \& Fidell, L. S. (2012). Using Multivariate Statistics. Person Education.

Ullrich-French, S., González, H. J., Dolores, M., \& Montesinos, H. (2017). Validity evidence for the adaptation of the State Mindfulness Scale for Physical Activity (SMS-PA) in Spanish youth. Psicothema, 29(1), 119-125. https://doi.org/10.7334/psicothema2016.204

Vallerand, R. J. (2015). The Psychology of Passion: A Dualistic Model. Oxford University Press.

Yan, Z. (2008). Psychological factors related to reasons for exercise: A comparative study between chinese and american college students [Unpublished master's thesis] Bowling Green State University, Ohio. https://etd.ohiolink.edu/!etd.send_file?accession=bgsu1213642219\&dispos ition $=$ inline

Yan, Z., Berger, B., Tobar, D., \& Cardinal, B. J. (2014). Reasons for Exercise Behavior Among American and Chinese College Women. Women in Sport and Physical Activity Journal, 22(1), 18-23. https://doi.org/10.1123/wspaj.2014-0005

recebido em setembro de 2019 aprovado em agosto de 2020

\section{Sobre o autor}

Evandro Morais Peixoto, docente do Programa de Pós-Graduação Stricto Sensu em Psicologia da Universidade São Francisco - USF. Doutor em Psicologia pela Pontifícia Universidade Católica de Campinas PUCC. Membro do GT Avaliação Psicológica em Psicologia Positiva e Criatividade na ANPEPP.

\section{Como citar este artigo}

Peixoto, E. V. (2021). Exercício Físico: Compreendendo as Razões para Prática e seus Desfechos Psicológicos Positivos. Avaliação Psicológica, 20(1), 52-60. http://dx.doi.org/10.15689/ap.2021.2001.18940.06 